
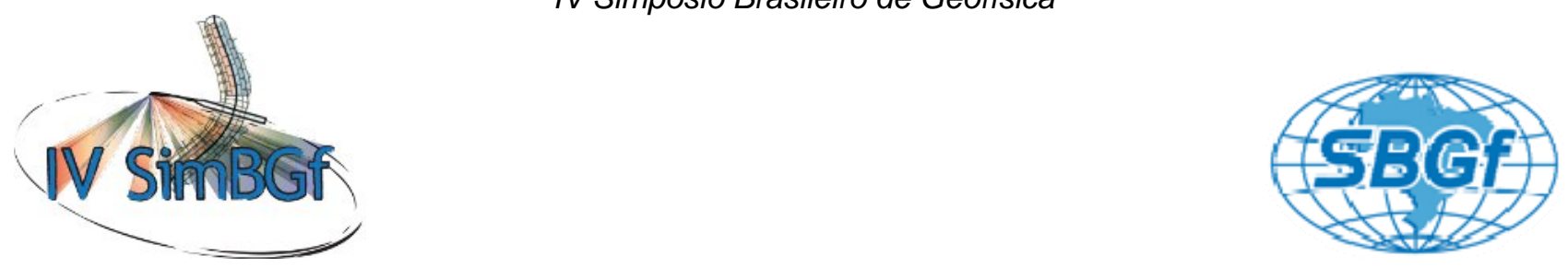

\title{
Identificação preliminar de calcita e dolomita em imagens termais na região de Campos Belos, GO.
}

Ana Raíssa Amorim dos Santos, Gustavo Macedo de Mello Baptista, Paulo Roberto Meneses, Instituto de Geociências Universidade de Brasília

Copyright 2010, SBGf - Sociedade Brasileira de Geofísica

Este texto foi preparado para a apresentação no IV Simpósio Brasileiro de Geofísica, Brasília, 14 a 17 de novembro de 2010. Seu conteúdo foi revisado pelo Comitê Técnico do IV SimBGf, mas não necessariamente representa a opinião da SBGf ou de seus associados. É proibida a reprodução total ou parcial deste material para propósitos comerciais sem prévia autorização da SBGf.

\section{Resumo}

O presente estudo preliminar buscou investigar as feições espectrais da calcita e da dolomita no contexto geológico de Campos Belos, GO, por meio de dados de emissividade obtidos das imagens termais do sensor ASTER. Para tal, utilizou a técnica de ajuste, por mínimos quadrados. de feição espectral SFF, ou Spectral Feature Fitting. Como endmembers utilizou-se os espectros de reflectância reamostrados para emissividade da biblioteca espectral da Universidade Johns Hopkins para minerais na faixa de 2,08 a $25 \mu \mathrm{m}$. Apesar de utilizar espectros de laboratório como referência, o algoritmo não separou bem os dois minerais na região estudada, pois os dados de intensidade de feição para um mineral correspondeu aos de erro médio quadrático do outro mineral. Além disso, as áreas consideradas pelo algoritmo como de dolomita apresentaram feições mais semelhantes aos da apatita, também presente na área. Novos estudos na faixa dos espectros emitidos devem ser incentivados para a separação desses minerais.

\section{Introdução}

Desde seu lançamento em 1999, o sensor ASTER (Advanced Spaceborne Thermal Emission and Reflection Radiometer), tem a sua potencialidade já bem documentada em diversos trabalhos acerca do monitoramento e exploração dos recursos naturais terrestres (e.g., DUCART, 2003; AUGUSTO et al., 2006). As técnicas de classificação usadas para identificação mineral por meio de sensoriamento remoto hiperespectral envolvem a comparação da similaridade dos espectros de reflectância extraídos diretamente das imagens com espectros de referência dos materiais testados em laboratório (CLARK et al., 1990; KRUSE et al., 1993; BAPTISTA et al., 1998).

Em geral, o uso dessas técnicas de identificação de minerais tem sido feito para análise de imagens no campo do espectro óptico refletido. A proposta deste presente artigo é realizar tal análise pra imagens na faixa do espectro emitido à temperatura natural dos alvos de superfície, ou seja, de 8 a $12 \mu \mathrm{m}$ ou infravermelho termal. A área estudada localiza-se a norte da cidade de Campos Belos, Goiás, próxima à divisa com o estado do Tocantins, e foi escolhida devido ao prévio conhecimento de sua geologia, condição necessária para que houvesse a validação do método utilizado neste trabalho.

$\mathrm{Na}$ região nota-se a presença de rochas pertencentes à sequência pelito-carbonatada do Grupo Bambuí (DARDENNE, 1978), por isso a escolha de calcita e dolomita como os minerais estudados.

\section{Material e Métodos}

Os dados de reflectância usados provem da Biblioteca Espectral da Universidade Johns Hopkins para minerais $(2,08$ a $25 \mu \mathrm{m})$, e foi utilizada a Lei de Kirchhoff's para transformar os dados de reflectância em emissividade por meio do módulo Spectral Math (Salisbury et al, 1994):

$E-1-\mathbf{R}$

Onde $\mathrm{E}$ corresponde a emissividade e $\mathrm{R}$, a reflectância. Após esse processo foi realizada a reamostragem da biblioteca espectral de emissividade, utilizando o software ENVI $^{\circledR}$ por meio do módulo Spectral Library Resampling, para as funções dos detectores do sensor TIR do ASTER (8 a $12 \mu \mathrm{m})$.

Adotou-se a cena ASTER sob demanda (AST_05) de emissividade no termal, já corrigida aos efeitos atmosféricos de absorção e espalhamento.

Selecionou-se um espectro de referência (ou endmembers) de calcita e um de dolomita reamostrados e utilizou-se o método de classificação Spectral Feature Fitting (CLARK et al., 1990).

No método SFF o espectro dos minerais selecionados como endmembers serve como um espectro "teste" que varre a imagem e, por meio de algoritmos, encontra os pixels de resposta semelhante ao de espectro teste (JUDD e STEINBERG, 2007).

O resultado desse processo são duas imagens separadas: a imagem scale que apresenta a medida da intensidade da feição espectral, e outra, RMS que representa o erro médio quadrático (BAPTISTA et al., 2007). Existe, ainda, a possibilidade de, ao dividir a imagem scale pela RMS, se obter a fit image, que é entendida como a probabilidade de o pixel apresentar o endmember.

Após a aplicação do SFF, foram usados filtros passabaixa $5 \times 5$, seguidos de expansão histogrâmica linear (Figura 1) para aumento de contraste, e por ultimo aplicou-se a tabela de cores rainbow. 


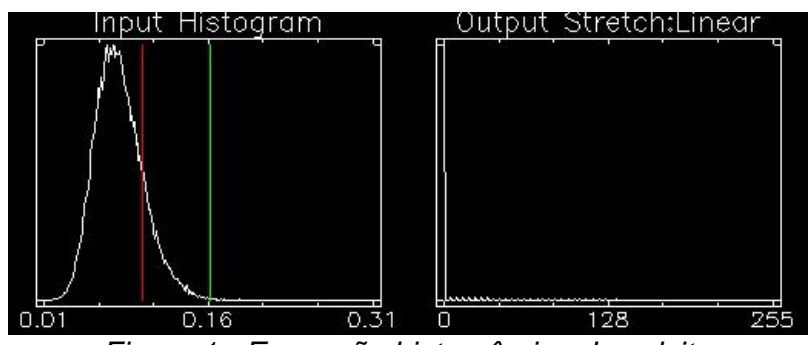

Figura 1 - Expansão histogrâmica da calcita.

Para comparação foram utilizados dois espectros da Biblioteca Espectral da Arizona State University - ASU que são obtidos em valores de emissividade na faixa de 5 a $45 \mu \mathrm{m}$, também reamostrados para as funções dos detectores do ASTER.

\section{Resultados}

Após a reamostragem feita dos dados da Biblioteca da Universidade de Johns Hopkins foram obtidos dois espectros relacionados aos endmembers calcita e dolomita (Figura 2).

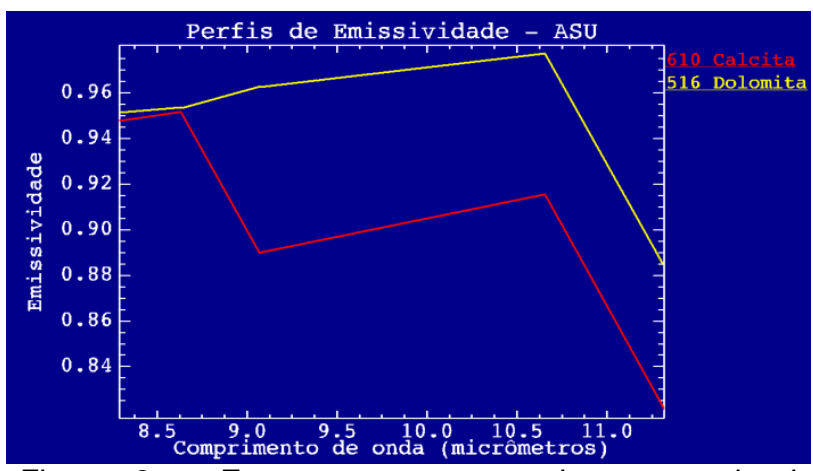

Figura 2 - Espectros testes usados, a partir da reamostragem dos dados da Universidade Johns Hopkins.

A partir desses espectros é realizada a SFF, formando as imagens scale e RMS, posteriormente trabalhadas para melhor visualização dos resultados.

Nos resultados da imagem scale (Figura 3) e RMS (Figura 4) para calcita onde as áreas em vermelho são as de maior resposta espectral.

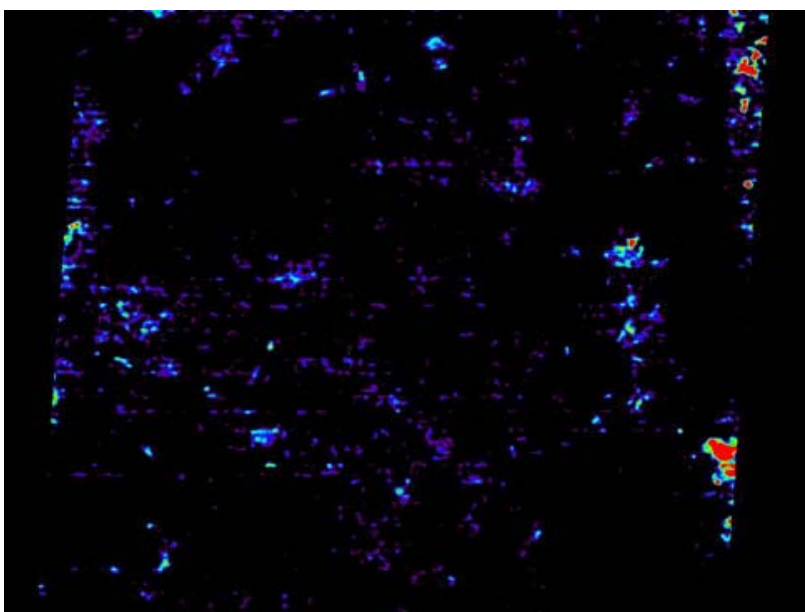

Figura 3 - Imagem scale para calcita.

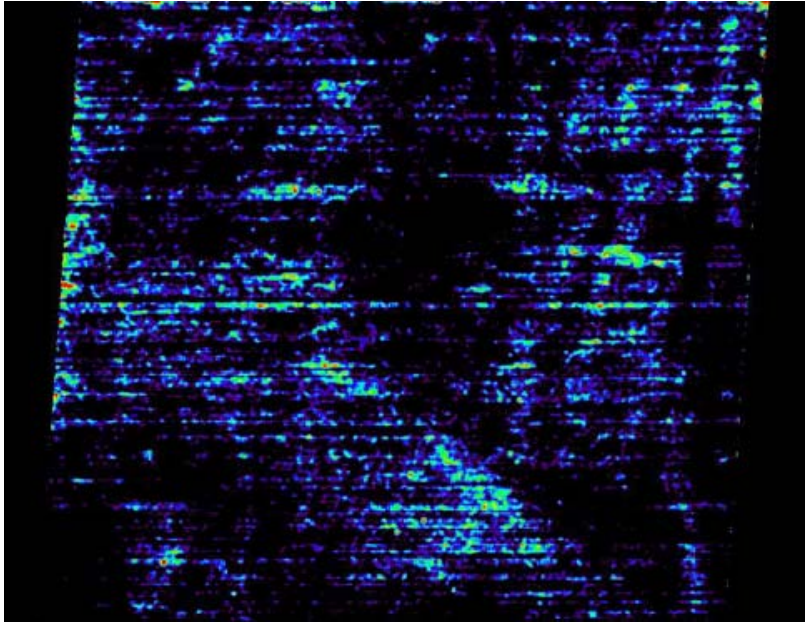

Figura 4 - Imagem RMS para calcita.

Nos resultados da imagem scale (Figura 5) e RMS (Figura 6) para dolomita onde as áreas em vermelho são as de maior resposta espectral.

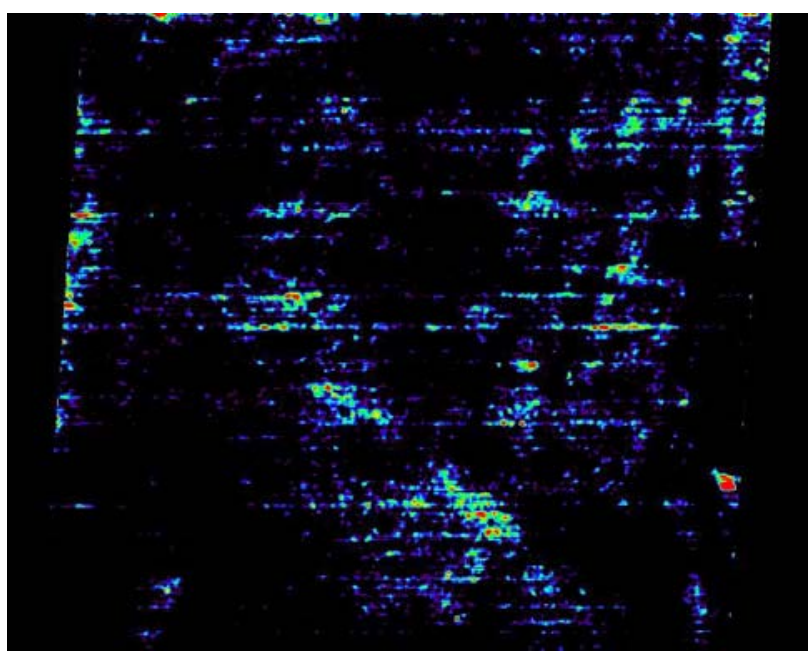

Figura 5 - Imagem scale para dolomita. 


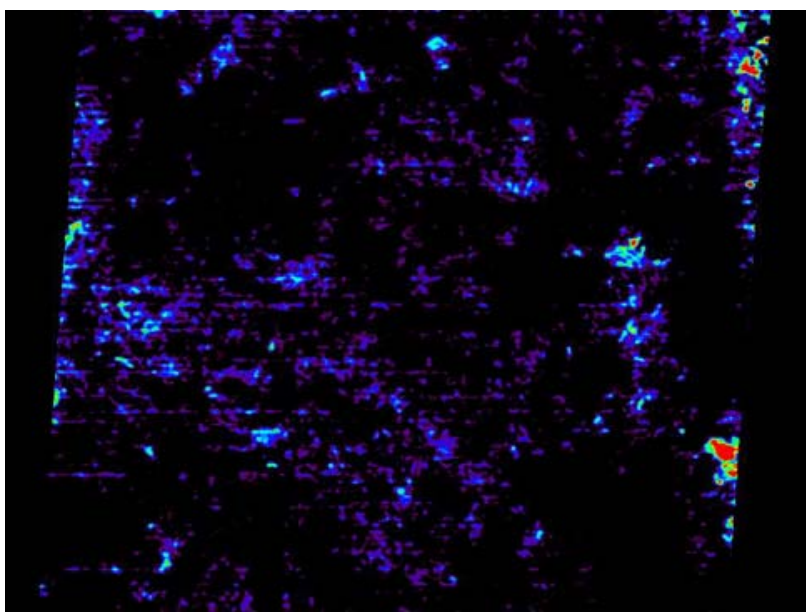

Figura 6 - Imagem RMS para dolomita.

\section{Discussão e Conclusões}

Analisando as imagens geradas, nota-se que a imagem scale de dolomita tem suas áreas de maior resposta espectral coincidentes com as áreas de maior erro da imagem RMS da calcita.

A partir dos resultados obtidos nas imagens SSF, foram escolhidos dois pixels de resposta espectral satisfatória, presentes nas imagens scale, sendo um para calcita (Figura 7) e outro para dolomita (Figura 8).

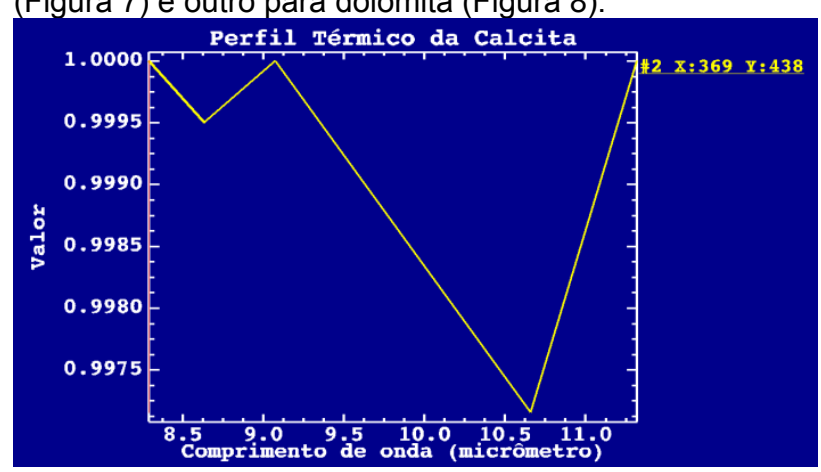

Figura 7 - Perfil térmico de um pixel onde houve resposta positiva para calcita na imagem scale.

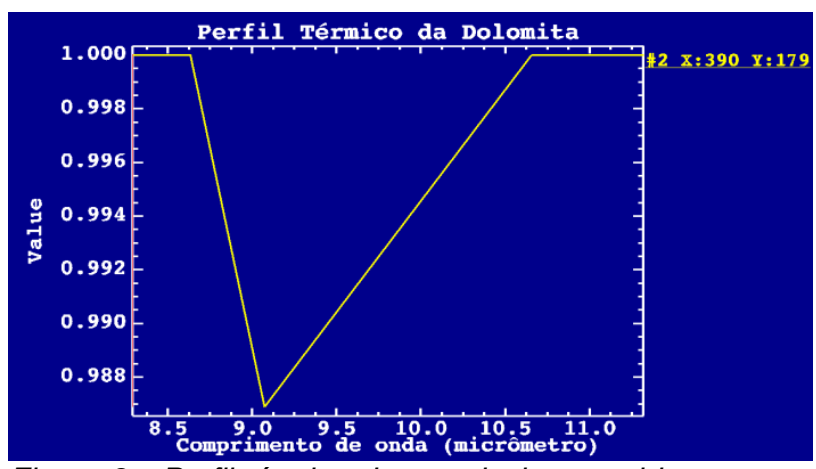

Figura 8 - Perfil térmico de um pixel no qual houve uma resposta positiva para dolomita na imagem scale.

Comparando-se o perfil térmico de resposta da imagem com o perfil de emissividade dos endmembers (Figura 2) usados como espectros testes, nota-se a pouca semelhança entre os espectros, o que leva a conclusão de que, para esta região estudada, a tentativa de separação desses minerais não foi satisfatória.

Isso pode ser devido ao fato de que, analisando espectros de calcita e dolomita obtidos nas áreas mapeadas pelo SFF, com o contínuo espectral removido (Figura 9), percebe-se que a resposta do pixel de dolomita apresenta mais semelhanças com o espectro de calcita do que o próprio pixel de calcita, o que justifica a imagem scale de dolomita ter suas maiores respostas em áreas coincidentes com a imagem RMS de calcita. Além disso, o espectro obtido na imagem nas áreas classificadas como dolomita, apresentam-se muito similares aos da apatita também encontrada na área de Campos Belos.

Como o sensor SWIR deixou de funcionar em abril de 2008 devido ao superaquecimento ocasionado pelo vazamento de radiação, conhecido como crosstalk, é importante o desenvolvimento de métodos de investigação mineralógica. Portanto, novos contextos que apresentem esses dois minerais devem ser investigados como forma de tentar separá-los na faixa do espectro emitido.

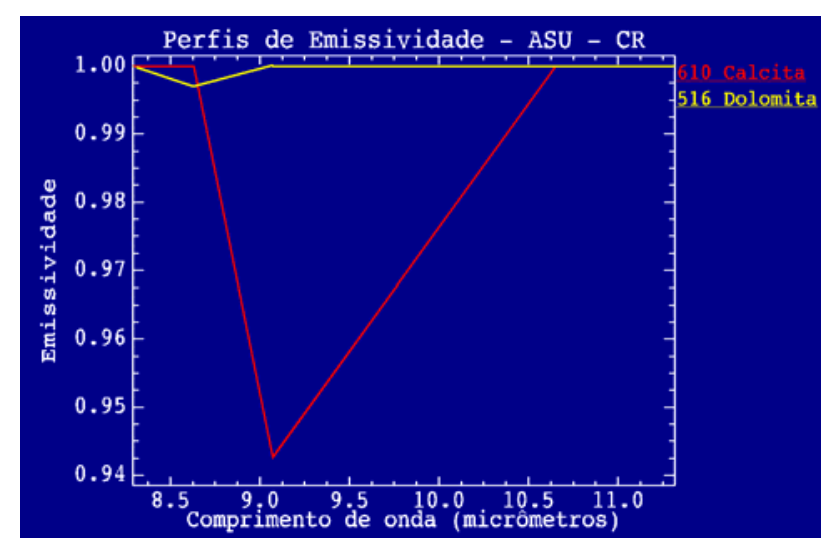

Figura 9 - Perfis de emissividade dos endmembers obtidos na imagem AST_05 após a aplicação do algoritmo SFF com a remoção do contínuo espectral.

\section{Referências}

Augusto, V. A.; Souza Filho, C. R.; Almeida-Filho, R. Processamento de imagens ASTER para detecção de áreas com microexsudações de hidrocarbonetos. In: Congresso Brasileiro de Geologia, 43. 2006, Aracaju, Anais..., p. 153, 2006.

Baptista, G.M.M.; Martins, E.S.; Madeira Netto, J.S.; Carvalho Júnior, O.A.; Meneses, P.R. Use of AVIRIS data for mineralogical mapping in tropical soils, in the district of São João D'Aliança, Goiás. Summaries of The Seventh Annual JPL Airborne Earth Science Workshop. Pasadena: Jet Propulsion Laboratory, 1998. v. 97. p. 3342.

Baptista, G. M. M.; Corrêa, R. S.; Torres, M. G., Bias, E. S.; Resende, M. G.; Ribeiro, R. J. C.; Silva, D. J. 
Identificação de relação mineral Caulinita/Gibbsita em solos tropicais, por meio dos dados SWIR do sensor ASTER. In Simpósio Brasileiro de sensoriamento Remoto, 13. Florianópolis, Anais..., p. 7043-7050,2007.

Clark, R. N.; Gallagher, A. J.; Swayze, G. A. Material absorption band depth mapping of imaging spectrometer data using a complete band shape least-squares fit with library reference spectra. In: Airborne Visible/Infrared Imaging Spectrometer (Aviris) Workshop, 2., 1990, Pasadena. Proceedings... Pasadena: Jet Propulsion Laboratory. p. 176-186. (Publication, 9054), 1990.

Dardenne, M.A. Síntese sobre a estratigrafia do Grupo Bambui no Brasil Central. SBG, Congr. Bras. Geol., 30, Recife. Anais, v. 2, p. 597-610, 1978.

Ducart, D. F. Caracterização de alteração hidrotermal associada às ocorrências auríferas de Los Menucos, Argentina, por meio de técnicas de sensoriamento remoto e espectroscopia de reflectância. 2004. 86 p. Dissertação de mestrado. Instituto de Geociências, Universidade Estadual de Campinas, Campinas-SP, 2004.

Judd, C.; Steinberg, S. Mapping submerged macrophytes: using multi-range spectral feature fitting to map submerged eelgrass in a turbid estuary. ASPRS Annual Conference 2007, Tampa, Florida, p.0033-0038, 2007.

Kruse, F. A.; Lefkoff, A. B.; Boardman, J. B.; Heidebrecht, K.B.; Shapiro, A. T.; Barloon, P.J.; Goetz, A. F.H. The Spectral Image Processing Systems (SIPS) - Interactive Vizualization and Analysis of Imageing spectrometer Data. Remote Sensing of Environment, v. 44, p. 145163,

Salisbury, J. W., Wald, A., and D'Aria, D. M., 1994, Thermal-infrared remote sensing and Kirchhoff's law 1. Laboratory measurements: Jour. of Geophysical Research, v. 99, p. 11,897-11,911. 